\title{
WP 35_12
}

\author{
Dragan Kukolj
}

University of Novi Sad, Serbia

Nikola Gradojevic

Lakehead University, Canada

The Rimini Centre for Economic Analysis (RCEA), Italy

Camillo Lento

Lakehead University, Canada

\section{IMPROVING NON-PARAMETRIC OPTION Pricing dURINg The FinanCial CRISIS}

\footnotetext{
Copyright belongs to the author. Small sections of the text, not exceeding three paragraphs, can be used provided proper acknowledgement is given.
}

The Rimini Centre for Economic Analysis (RCEA) was established in March 2007. RCEA is a private, nonprofit organization dedicated to independent research in Applied and Theoretical Economics and related fields. RCEA organizes seminars and workshops, sponsors a general interest journal The Review of Economic Analysis, and organizes a biennial conference: The Rimini Conference in Economics and Finance (RCEF) . The RCEA has a Canadian branch: The Rimini Centre for Economic Analysis in Canada (RCEACanada). Scientific work contributed by the RCEA Scholars is published in the RCEA Working Papers and Professional Report series.

The views expressed in this paper are those of the authors. No responsibility for them should be attributed to the Rimini Centre for Economic Analysis. 


\title{
Improving Non-parametric Option Pricing during the Financial Crisis
}

\author{
Dragan Kukolj, Senior Member, IEEE, Nikola Gradojevic, Camillo Lento
}

\begin{abstract}
Financial option prices have experienced excessive volatility in response to the recent economic and financial crisis. During the crisis periods, financial markets are, in general, subject to an abrupt regime shift which imposes a significant challenge to option pricing models. In this context, swiftly evolving markets and institutions require valuation models that are capable of recognizing and adapting to such changes. Both parametric and non-parametric pricing models have shown poor forecast ability for options traded in late 1987 and 2008. Surprisingly, the pricing inaccuracy was more pronounced for non-parametric models than for parametric models. To address this problem, we propose a modular neural network-fuzzy learning vector quantization (MNN-FLVQ) model that uses the Kohonen unsupervised learning and fuzzy clustering algorithms to classify the S\&P 500 stock market index options, and thereby detect a regime shift. The results for the 2008 financial crisis demonstrate that the MNN-FLVQ model is superior to the competing methods in regards to option pricing during regime shifts.
\end{abstract}

\section{INTRODUCTION}

In the summer of 2008, significant concerns mounted regarding the health of the U.S. financial markets and institutions. Subsequent events of September and October of that year resulted in an unprecedented financial crisis marked by excess volatility, strong risk aversion and large daily stock price swings. As option price changes tend to be an amplification of stock price movements, "tail events" in equity markets can induce extreme option price levels. Typically, put option premiums increase substantially as investors purchase insurance for their stock portfolios. Also, during market crises, high volatility increases the price of call options. These intense fluctuations lead to a market regime shift which makes it very challenging to select an appropriate option pricing model.

In response to these issues, the traditional Black-Scholes option pricing model [1] has been abandoned in favor of models that incorporate both stochastic volatility and jumps components [2][4]. However, jump diffusion models do not sufficiently describe the systematic variations in option

\footnotetext{
D. Kukolj thanks the Ministry of Education and Science of the Republic Serbia (Grant TR-32034) and the Secretary of Science and Technology Development of Vojvodina Province (Grant 114-451-2434/2011-01) that partially supported this work. N. Gradojevic gratefully acknowledges financial support from the Social Sciences and Humanities Research Council of Canada.

D. Kukolj is with the Faculty of Technical Sciences, Novi Sad, Serbia (e-mail: dragan.kukolj@rt-rk.com).

N. Gradojevic is with the Faculty of Business Administration, Lakehead University, Canada and with the Rimini Center for Economic Analysis, Italy. (phone: 1-807-343-8419; fax: 1-807-343-8443; e-mail: ngradoje@lakeheadu.ca).

C. Lento is with the Faculty of Business Administration, Lakehead University, Canada (e-mail: clento@lakeheadu.ca).
} 
prices. Accordingly, alternative models that include jumps to the volatility process have been developed.

Research concludes that stochastic volatility (SV) models are dominated by stochastic volatility random jump (SVJ) models in out-of-sample pricing exercises. In general, parametric option pricing literature reveals that the SV, SVJ, and stochastic interest rate (SI) models improve upon the basic Black-Scholes model with equity indices [5]. Essentially, a flexible distributional structure and the volatility variability property of the SV model control for the level of skewness and the excess kurtosis; however, these features are insufficient to explain discontinuous jumps and crashes. SVJ models present an extension of SV models as they internalize the jumps and crashes and account for the skewness and high kurtosis. This leads to more accurate short to medium term option pricing. For example the SI, SV and SVJ models have been shown to significantly improve the pricing accuracy of the Black-Scholes model for the medium to long term options, while they exhibit some moneyness-related biases for the short-term options [5].

The observed biases with parametric models have motivated the development of more complex semi-parametric and non-parametric option pricing models. For example, the out-of-sample performance of an artificial neural network (NN) model was compared to the SVJ, SI and SV parametric approaches for the S\&P 500 stock market index over the 1989-1991 data span [9]. The comparison concludes that the NN pricing model with the $\operatorname{GARCH}(1,1)$ volatility dominates all parametric models. Some other important contributions in this respect are the mixture of distributions model by Melick and Thomas [11] and the semi-parametric estimator by Ait-Sahalia and Lo [10]. These models have shown sizable improvements in option pricing accuracy compared to the Black-Scholes model; however, their out-of-sample pricing is inferior to non-parametric modular neural network (MNN) models [12]. Some recent non-parametric approaches also include the affine jump-diffusion models [14] and the normal inverse Gaussian models [13], [22]. More recently, fuzzy logic has been shown to be very useful for option pricing [15] and [16]. Finally, entropy-based option pricing models are a worthy, yet relatively unexplored, area [17], [18]. ${ }^{1}$

Typically, non-parametric models, or the so-called data-driven approaches, offer superior pricing performance relative to parametric models due to the restrictive cumulative normal functional shapes used by parametric benchmarks. In contrast, non-parametric models provide flexibility by allowing a variety of cumulative distributions such as the sigmoidal function [8], the generalized hyperbolic class [19] and a q-Gaussian [17]. These flexible functional forms effectively accommodate jumps, non-stationarity, and negative skewness and kurtosis. The success of non-

\footnotetext{
${ }^{1}$ Excellent reviews of the option pricing literature can be found in Garcia et al. [4] and Renault [21].
} 
parametric option pricing models can also be explained by their adaptive learning capabilities, considering that models such as NN are trained to generalize in the out-of-sample data [9].

Notwithstanding the effectiveness of non-parametric models, smoothing a curve and achieving stability in the out-of-sample performance can be a challenging task. This is particularly the case during the periods of market booms and crashes. In such situations, the very advantages of nonparametric models over their parametric counterparts may contribute to their poor out-of-sample performance. Specifically, when a non-parametric model is estimated (trained) in-sample, it is based on historical data, preferably from the recent history.

Suppose the current market is experiencing a crash. Option pricing in such an episode will be based on variables estimated from a different market regime. Put differently, a non-parametric model is expected to price options from the crisis regime based on the information (data) collected during stable market conditions. This regime shift may be detrimental to the out-of-sample performance of non-parametric methods. Therefore, a parsimonious parametric model that is a prespecified non-linearity whose shape does not depend on the in-sample data may be more appropriate. For example, out-of-sample pricing inaccuracy is found when the NN model was estimated based on the first two quarters and tested on the last quarter of 1987 [8]. Similar observations were reported for the 1987 regime shift [4], [12] and [16]. For instance, risk-neutral distributions recovered from option prices before and after the crash of 1987 are shown to be fundamentally different [2]. In the period before the crash, both the risk neutral and the actual distributions are roughly lognormal, whereas following the crash, the risk-neutral distribution becomes left-skewed and leptokurtic.

A possible explanation for the regime shift is a change in the risk aversion of an average investor. In the same vein, another interpretation of this structural change is based on jumps and learning [23]. The idea is that prior to the $1987 \mathrm{crash}$, investors attribute a very low probability to extreme jumps, but, after it takes place, the investors update their beliefs about the likelihood of future jumps, which increases the intensity of the market crash. This leads to a stark regime shift in the S\&P 500 index option prices.

This paper proposes a novel approach to the pricing of options during the late 2008 financial crisis regime shift. Our model combines an MNN model and the fuzzy learning vector quantization (FLVQ) algorithm to classify the S\&P 500 stock market index options across the maturity and moneyness ranges. The FLVQ algorithm relies on a Kohonen unsupervised NN and fuzzy logic cmeans clustering. This methodology produces a set of 'intelligent' boundaries between the modules of an MNN model where each module represents a particular market regime. Such classification enables a smoother transition from the training, pre-crisis data to the crisis regime and, thus, 
improves the model's generalization ability. As a result, the model exhibits outstanding out-ofsample pricing accuracy when compared to competing models that largely fail to account for the structural changes in last quarter of 2008. Our findings emphasize the pitfalls of employing heterogeneous data for training and testing of non-parametric models. Sometimes referred to as "spatial crosstalk" [24], this problem may lead to slow convergence and poor function approximation.

The structure of the article is as follows: Section II explains the MNN-FLVQ methodology; Section III explains the data and Section IV presents the results of our out-of-sample option pricing exercises. Section V concludes and suggests extensions of the current approach.

\section{MNN-FLVQ MODEL}

The basic idea of the MNN-FLVQ model is the fact that an arbitrarily complex model explaining a financial signal is a combination of mutually interlinked sub-models that process certain aspects (i.e., regimes) of a financial signal of interest. The FLVQ algorithm is used for classification of the regimes into clusters (or modules) that are then processed by the MNN model.

\section{A. MNN Pricing Model}

To explain the MNN part of the MNN-FLVQ model, first, we will assume that a function of two variables $c_{t}\left(c_{t}=\phi\left(x_{1 t}, x_{2 t}\right)+\varepsilon_{t}\right)$ is driven by different functions defined over known domains of $x_{l t}$ $(l=1,2)$, where $t$ denotes the time index. Namely, function $\phi\left(x_{1 t}, x_{2 t}\right)$ can be written as a linear combination of functions $c_{1 t}, c_{2 t}, \ldots, c_{M t}$ as follows [7], [25]:

$$
\begin{aligned}
\phi\left(x_{1 t}, x_{2 t}\right) & =w_{1} c_{1 t}\left(x_{1 t}, x_{2 t}\right)+\ldots+w_{M} c_{M t}\left(x_{1 t}, x_{2 t}\right) \\
& =\sum_{k=1}^{M} w_{k} c_{k t}\left(x_{1 t}, x_{2 t}\right)
\end{aligned}
$$

This function can be approximated by an MNN. A meaningful decomposition of the function $\phi\left(x_{1 t}, x_{2 t}\right)$ would be to approximate it locally by $M$ modules $c_{1 t}, c_{2 t}, \ldots, c_{M t}$ and then to sum the output of each module, while applying the appropriate weights $w_{1}, w_{2}, \ldots, w_{M}$. In this paper, we apply a FLVQ partitioning approach that sets only one $w_{i}=1$, while all other $w_{k}=0$ for $k \neq i, k=1, \ldots, M$. This can be viewed as a "crude partitioning" of the input space, i.e., the weights are redistributed to only one 
module at the time. In other words, after the FLVQ algorithm determines the membership of each input observation, there is no interaction among modules, i.e., the regimes are distinct and partial memberships are not considered [Fig. 1].

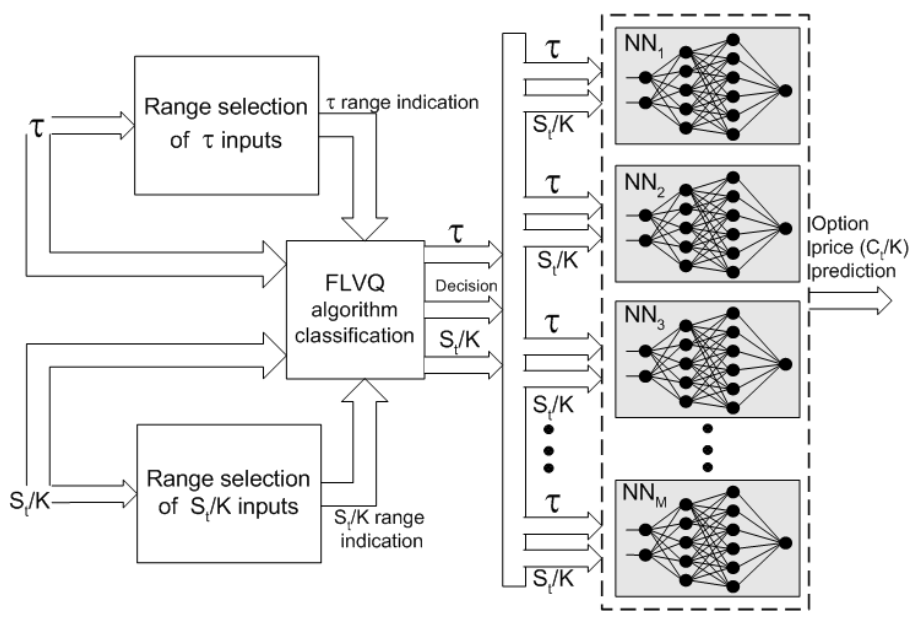

Fig. 1. MNN-FLVQ model architecture.

Notes: After the FLVQ algorithm determines the membership of each input observation, the predictions are generated by the MNN model that activates one of its neural network modules $\left(\mathrm{NN}_{1}, \ldots, \mathrm{NN}_{\mathrm{M}}\right)$.

We specify modules and weights using the stock-price-to-strike-price ratio $\left(S_{t} / K\right)$ and time-tomaturity $(\tau)$ criteria. The modules are feedforward (backpropagation) NNs and are in general estimated as

$$
\begin{gathered}
c_{k t}=g_{k}\left(\beta_{k 0}+\sum_{j=1}^{q_{k}} \beta_{k j} \psi_{k}\left(\alpha_{k j 0}+\sum_{i=1}^{s} \alpha_{k i j} x_{i t}\right)\right), \\
k=1, \ldots, M
\end{gathered}
$$

In our case, $s=4(i=1,2,3,4)$ and $q_{k}$ is the number of hidden nodes for the $k^{\text {th }}$ module. The single hidden and the output layers of the modules are characterized by two flexible classes of nonlinearities: $\psi_{k}$ and $g_{k}$, respectively. The backpropagation learning algorithm requires continuous differentiable non-linearities. The types used in this paper are the sigmoid logistic or hyperbolic tangent functions in the hidden layer, and the linear function in the output layer. $\alpha_{k i j}$ and $\beta_{k j}$ denote 
appropriate connection weights between the adjacent layers for the modules. Subscripts 0 for $\alpha$ and $\beta$ stand for NN biases.

The option pricing formula for the S\&P 500 index call options is defined as in [8], with two additional inputs:

$$
C_{t}=\phi\left(S_{t}, K, r_{t}, \sigma_{t}, \tau\right),(3)
$$

where $C_{t}$ is the call option price, $S_{t}$ is the price of the underlying asset, $K$ is the strike price, $\sigma_{t}$ is the volatility of the underlying asset, $r_{t}$ is the interest rate, and $\tau$ is the time to maturity (number of days). Assuming the homogeneity of degree one of the pricing function $\phi$ with respect to $S_{t}$ and $K$, one can write the option pricing function as follows:

$$
\underbrace{\frac{C_{t}}{K}}_{c_{t}}=f(\underbrace{\frac{S_{t}}{K}}_{x_{1}}, 1, \underbrace{r_{t}}_{x_{2}}, \underbrace{\sigma_{t}}_{x_{3}}, \underset{x_{4}}{\tau})=f\left(x_{1}, x_{2}, x_{3}, x_{4}\right)
$$

There is also a technical reason for dividing by the strike price $(K)$ since the process $S_{t}$ is nonstationary while the variable $S_{t} / K$ is stationary. This paper uses (4) for the non-parametric (MNN) model estimation.

\section{B. FLVQ Classification Algorithm}

The FLVQ algorithm belongs to the class of batch unsupervised learning methods [26]. The FLVQ algorithm represents a combination of the weight adaptation rule used in the Kohonen unsupervised neural network and the fuzzy set membership function proposed by the batch clustering fuzzy cmeans (FCM) algorithm. In comparison to similar clustering methods, the main advantages of the FLVQ algorithm are: 1) smaller number of input (user-defined) parameters is required, 2) less frequently trapped in local minima, and 3) final solution is not affected by the order of the input data sequence. The clustering process of the FLVQ algorithm is based on the assumption that each data class may contain several clusters defined by their representative points called prototypes. In general, the algorithm contains two phases: the initialization of the prototype coordinates of the clusters (prototype generation) and the improvement of classification error by adapting the prototypes' coordinates (prototype adaptation).

The FLVQ is a self-organizational, non-sequential and competitive clustering algorithm. Using the training data, after the initial number of classes and prototypes are defined, for each input 
observation from the training set, the density of distribution of other input data in its neighborhood is determined from the FCM algorithm. The output of this algorithm represents the coordinates of the data with the highest density, which are adopted as the initial values of the prototypes.

Classes that should be extended with new prototypes are identified in an iterative process. Those classes are considered to have the largest number of misclassified data. In other words, if an input pattern has the nearest prototype from the same class it is correctly classified and vice-versa. In each iteration, a partition matrix $U$ of the current prototypes is calculated by:

$$
\begin{aligned}
& u_{i, j}=\frac{\left(\frac{1}{d\left(x_{j}(e), C_{i}(e)\right)}\right)^{\frac{1}{m(e)-1}}}{\sum_{k=1}^{K}\left(\frac{1}{d\left(x_{j}(e), C_{k}(e)\right)}\right)^{\frac{1}{m(e)-1}},} \\
& i=1, \ldots K, j=1, \ldots, N .
\end{aligned}
$$

where $N$ is the number of observations in the training data, $K$ is total number of prototypes, $x_{j}$ is the $j^{\text {th }}$ input pattern from the training set, $C$ is the matrix of prototypes coordinates, $d\left(x_{j}(e), C_{k}(e)\right)$ is the Euclidean distance between $j^{\text {th }}$ input pattern and the $k^{\text {th }}$ prototype in each training (iteration) epoch $e$ and the weighting exponent $m(e)$ is called the "degree of fuzziness" in epoch $e$.

The degree of fuzziness $m=m(e)$ is monotonically decreasing with respect to the processing time $e$, i.e., the number of training epochs:

$$
m(e)=m_{0}-\frac{e\left(m_{0}-m_{f}\right)}{e_{\max }} .
$$

where $m_{0}$ and $m_{f}$ are the initial and final values of the degree of fuzziness $m$, whose values are limited by the heuristically determined constraint: $7>m_{0}>m_{f}>1.1$. Reference [26] shows that this range is appropriate for a monotonically decreasing FLVQ algorithm. More specifically, these limits prevent numerical instability that may arise when values of $m$ are close to 1 or are very large (i.e., when $m$ tends to infinity). Parameter $e_{\max }$ determines the maximum number of epochs.

The coordinates of prototypes are modified using FLVQ learning rule given by following expression: 


$$
C_{i}(e+1)=\sum_{j=1}^{N} \alpha_{i, j}(e) \cdot x_{j}(e), i=1, \ldots, K
$$

where $\alpha_{i, j}(e)$ is the learning rate of the $i^{\text {th }}$ prototype in epoch $e$. This learning factor is set by:

$$
\alpha_{i, j}(e)=\frac{\left(u_{i, j}(e)\right)^{m(e)}}{\sum_{j=1}^{N}\left(u_{i, j}(e)\right)^{m(e)}},
$$

Equations (5)-(8) are repeated until the given number of prototypes $K$ is reached.

Finally, in the prototype adaptation phase the prototypes' coordinates are modified. The key parts of the algorithm are (7) and (8), where the elements of the partition matrix $U$ and the coordinates of the prototypes $C_{i}$ are updated. Based on the distances among all input patterns and the current position of the prototypes, new coordinates of the prototypes are calculated in each iteration. The parameter $m(e)$ and current epoch $e$ determine the prototype's neighborhood, while only patterns belonging to same class are considered. After this step, the total number of prototypes, as well as their number per each class are not changed. In the end, after $e_{\max }$ iterations, the best prototype coordinates, i.e., the prototypes with the highest number of correctly classified patterns are recorded and assigned to the classes.

\section{FLVQ Parameters}

We experimented with training data from the first (last) two quarters of 2008 and the validation data from the third (second) quarter representing the trial testing data. The most accurate pricing for the third (second) quarter data was obtained by using ten classes and fifty prototypes (with allowed maximum of 6 prototypes per class). These input parameters are then used in pricing the fourth (first) quarter options (true out-of-sample data). The initial and final values of the degree of fuzziness are $m(0)=1.1$ and $m(0)=2.2$, respectively.

\section{DATA}

The options data for 2008 were provided by DeltaNeutral and represent the daily S\&P 500 index European call option prices, taken from the Chicago Board Options Exchange. Call options across different strike prices and maturities are considered. Being one of the deepest and the most liquid option markets in the United States, the S\&P 500 index option market is sufficiently close to the 
theoretical setting of the Black-Scholes model. Options with zero volume are not used in the estimation. The risk-free interest rate $(r)$ is approximated by the monthly yield of the U.S. Treasury bills. The implied volatility $\left(\sigma_{I}\right)$ is a proprietary mean estimate provided by DeltaNeutral.

The data for each year are divided into three parts: first (last) two quarters (estimation data), third (second) quarter (validation data) and fourth (first) quarter (testing data). Our first exercise prices options on the fourth quarter of the year that includes the market crisis periods. The second pricing exercise focuses on the performance of the models in the first quarter of each year that represents the out-of-sample data. To reduce the size of the data set for 2008 , we also eliminated options with low volume (that traded below 100 contracts on a given day), and focused only on the close to atthe-money options (with strike prices between $95 \%$ and $105 \%$ of the underlying S\&P 500 index). This resulted in 14838 observations of which 3904 were in the first quarter, 4572 were in the second quarter, 4088 were in the third quarter and 2274 were in the fourth quarter of 2008.

The out-of-sample pricing performance of the MNN-FLVQ model is first compared to the wellknown benchmark - the Black-Scholes model. This model has proved to be a good pricing tool during the financial crisis of 1987 [8]. The Black-Scholes call prices $\left(C_{t}\right)$ are computed using the standard formula

$$
\begin{aligned}
& C_{t}=S_{t} \mathcal{N}(d)-K e^{-r \tau} \mathcal{N}(d-\sigma \sqrt{\tau}) \\
& \text { where } \quad d=\frac{\ln \left(S_{t} / K\right)+\left(r+0.5 \sigma^{2}\right) \tau}{\sigma \sqrt{\tau}},
\end{aligned}
$$

where $X$ is the cumulative normal distribution, $S_{t}$ is the price of the underlying asset, $K$ is the strike price, $\tau$ is the time to maturity, $r$ is the risk-free interest rate, and $\sigma$ is the volatility of the underlying asset's continuously-compounded returns. The risk-free rate is approximated using the monthly yield of U.S. Treasury bills.

The statistical significance of the difference in the out-of-sample (testing set) performance of alternative models is tested using the Diebold-Mariano test [27]. We test the null hypothesis that there is no difference in the mean-squared prediction error (MSPE) of the two alternative models. The Diebold-Mariano test statistic for the equivalence of forecast errors is given by

$$
D M=\frac{\frac{1}{M} \sum_{t=1}^{M} d_{t}}{\sqrt{\frac{2 \pi f(0)}{M}}},
$$


where $\mathrm{M}$ is the testing set size and $f(0)$ is the spectral density of $d_{t}$ (the forecast error is defined as the difference between the actual and the forecasted output value) at frequency zero. Diebold and Mariano show that DM is asymptotically distributed in a $N(0,1)$ distribution.

\section{ESTIMATION RESULTS}

The out-of-sample pricing performance of the MNN-FLVQ is evaluated on the last quarter (Q4) and first quarter of 2008 (Q1). The options are also priced by using the competing models: Garcia and Gençay's [6] feedforward NN model with the hint (NN with hint), the MNN model [12], and the Black-Scholes (BS) model [1]. The results for Q4 reveal that the MNN-FLVQ model statistically improves upon the BS model (Table I). Nonetheless, it is worthwhile to note that the BS model is remarkably accurate during the crisis period relative to the competing non-parametric methods. As expected, in the Q1 of 2008, the pricing ability of all approaches improves substantially. In particular, all three NN-based models dominate the BS model. The NN with the hint model is the most accurate. The statistical significance of the pricing improvements is confirmed by the large negative values of the DM statistics in the last row of Table I.

TABLE I.

PREDICTION PERFORMANCE OF THE COMPETING OPTION PRICING MODELS IN 2008

\begin{tabular}{|c|c|c|c|c|}
\hline & BS & $\begin{array}{c}\text { NN with } \\
\text { hint } \\
(\mathrm{DM})\end{array}$ & $\begin{array}{l}\text { MNN } \\
(\mathrm{DM})\end{array}$ & $\begin{array}{l}\text { MNN- } \\
\text { FLVQ } \\
(\mathrm{DM}) \\
\end{array}$ \\
\hline \multicolumn{5}{|l|}{$M A P E:$} \\
\hline Q4 & 0.5414 & 6.2910 & 0.3317 & 0.3304 \\
\hline Q1 & 0.1382 & 0.6807 & 0.0940 & 0.0924 \\
\hline \multicolumn{5}{|l|}{ MSPE: } \\
\hline Q4 & $3.05 \times 10^{-4}$ & $\begin{array}{c}17.3 \times 10^{-4} \\
(4.54)\end{array}$ & $\begin{array}{c}3.51 \times 10^{-4} \\
(0.33)\end{array}$ & $\begin{array}{r}\mathbf{1 . 8 8} \times \mathbf{1 0}^{-4} \\
(-1.61)\end{array}$ \\
\hline Q1 & $1.50 \times 10^{-4}$ & $\begin{array}{c}\mathbf{4 . 1 7} \times 10^{-5} \\
(-6.68)\end{array}$ & $\begin{array}{c}1.08 \times 10^{-4} \\
(-1.71)\end{array}$ & $\begin{array}{r}1.05 \times 10^{-4} \\
(-2.04)\end{array}$ \\
\hline
\end{tabular}

Notes: This table reports the out-of-sample average mean-absolute percentage error $(\overline{\overline{M A P E}})$ and the average meansquared prediction error ( $\overline{M S P E}$ ) of Garcia and Gençay's [6] feedforward neural network model with the hint (NN with hint), modular neural network model [12], modular neural network-fuzzy learning vector quantization model (MNNFLVQ) and the Black-Scholes (BS) model [1]. The average error figures are presented as averages across ten different random training seeds. The pricing error for a non-parametric model with four inputs $\left(S_{t} / K, \tau, r, \sigma\right)$ was calculated. "Q 1 ” ("Q4") denotes that the S\&P 500 Index call options were priced in the first (fourth) quarter of 2008 that was kept as out- 
of-sample observations. DM denotes the Diebold and Mariano (1995) test statistic. This test is used to assess the statistical significance of the MSPE forecast gains of each non-parametric model relative to the BS model. The minimum pricing error in each out-of-sample period is bolded.

We conclude that the regime shift which took place in late 2008 limits learning and generalization abilities of non-parametric models and results in pricing inaccuracy. However, the 'intelligent clustering' feature of the MNN-FLVQ model overcomes the problem that arises in non-parametric option pricing. Interestingly, being a pre-specified non-linearity, the BS model appears to be less sensitive to a regime shift than the NN models. This paper argues that the explanation for the success of the MNN-FLVQ model lies in its ability to decompose the option pricing function into a number of simpler sub-models (regimes) across moneyness and maturity ranges. These sub-models are estimated individually and each becomes activated when identified during the out-of-sample testing phase.

To illustrate the accuracy of the MNN-FLVQ model, the out-of-sample predictions of $c_{t}$ from (4) and the actual $\left(C_{t} / K\right)$ data for the last quarter of 2008 are plotted in Fig. 2. Despite the occasional extreme volatility outbursts in $c_{t}$ over the testing sample, the estimates follow the actual prices very closely.

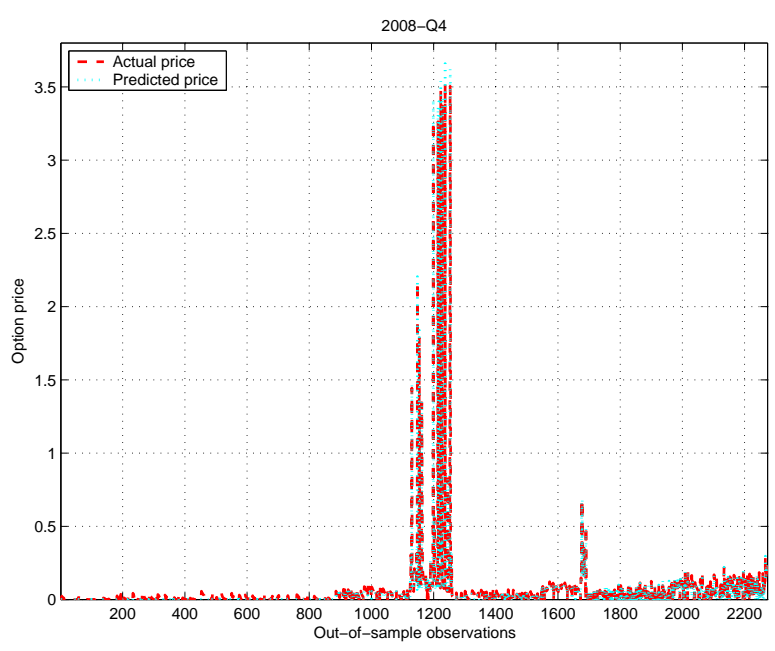

Fig. 2. Data and option prices estimated by the MNN-FLVQ model.

Notes: Out-of-sample predictions of $c_{t}$ (cyan, dotted line) and the actual data (red, dashed line) are plotted for the last quarter of 2008. First, the MNN-FLVQ model is estimated and validated using the data from the first three quarters of the year and, then, 2274 out-of-sample estimates of $c_{t}$ are generated. 


\section{CONCLUSIONS}

This paper proposes a novel methodology to price S\&P 500 Index options that are subject to a regime shift, such as options during the 2008 financial crisis. The importance of our work lies in its potential applicability to any other time-series or cross-sectional data that contain multiple regimes or structural breaks. In finance and economics, the variables of interest may include exchange rates, equity prices, interest rates and derivative prices. Therefore, with this contribution, we hope to motivate further research in this area that holds valuable implications for financial risk management and government policy.

There are two possible extensions of the MNN-FLVQ model that we plan to pursue in our future research. The first one concerns utilizing more complex clustering and classification methodologies such as Gaussian mixture models, genetic algorithms and ant colony optimization. Hence, improving the ability of the model to differentiate among heterogeneous option types (or market regimes) represents the key future research direction. For this purpose, in addition to the two present classification criteria (moneyness and maturity), the model can also be provided with more option-specific information. For instance, the interest rate criterion that relies on risk-free interest rates that match the maturity of individual options could be introduced. Likewise, option open interest might represent another informative variable for detecting regime shifts.

\section{REFERENCES}

[1] F. Black, and M. Scholes, "The pricing of options and corporate liabilities," Journal of Political Economy, vol. 81, pp. 637-659, 1973.

[2] D. Bates, "Post-'87 crash fears in S\&P 500 futures options," Journal of Econometrics, Vol. 94, pp. 181-238, 2000.

[3] J. Pan, "The jump-risk premia implicit in options: Evidence from an integrated time-series study," Journal of Financial Economics, Vol. 63, pp. 3-50, 2002.

[4] B. Eraker, "Do stock prices and volatility jump? Reconciling evidence from spot and option prices," Journal of Finance, Vol. 59(3), pp. 1367-1403, 2004.

[5] G. Bakshi, C. Cao and Z. Chen, "Empirical performance of alternative option pricing models," Journal of Finance, Vol. 52(5), pp. 2003-49, 1997.

[6] R. Garcia, E. Ghysels and E. Renault, "The econometrics of option pricing", in Handbook of Financial Econometrics, Y. Aït-Sahalia and L. P. Hansen, Eds., Amsterdam: Elsevier-North Holland, 2010. 
[7] D. Kukolj, "Design of adaptive Takagi-Sugeno-Kang fuzzy model," Applied Soft Computing, Vol. 2(2), pp. 89-103, 2002.

[8] R. Garcia, and R. Gençay, "Pricing and hedging derivative securities with neural networks and a homogeneity hint," Journal of Econometrics, Vol. 94(1-2), pp. 93-115, 2000.

[9] R. Gençay, and R. Gibson, "Pricing model risk for European-style stock index options," IEEE Transactions on Neural Networks, Vol. 18(1), pp.193-202, 2007.

[10] Y. Aït-Sahalia, and A.W. Lo, "Nonparametric estimation of state-price densities implicit in financial asset prices," Journal of Finance, Vol. 53, pp.499-547, 1998.

[11] W. R. Melick and C. P. Thomas, "Recovering an asset's implied PDF from option prices: An application to crude oil during the gulf crisis," Journal of Financial and Quantitative Analysis, Vol. 32(1), pp. 91-115, 1997.

[12] N. Gradojevic, R. Gençay, and D. Kukolj, "Option pricing with modular neural networks," IEEE Transactions on Neural Networks, Vol. 20(4), pp. 626-637, 2009.

[13] A. Erikkson, E. Ghysels, and F. Wang, "The normal inverse Gaussian distribution and the pricing of derivatives," Journal of Derivatives, Vol. 16, pp. 23-38, 2009.

[14] P. Carr, and L. Wu, "Time-changed Lévy processes and option pricing," Journal of Financial Economics, Vol. 71, pp. 113-141, 2004.

[15] E. Agliardi, and R. Agliardi, "Fuzzy defaultable bonds," Fuzzy Sets and Systems, Vol. 160(18), pp. 2597-2607, 2009.

[16] N. Gradojevic, and D. Kukolj, "Parametric option pricing: A divide-and-conquer approach," Physica D, Vol. 240(19), pp. 1528-1535, 2011.

[17] L. Borland, "A theory of non-Gaussian option pricing," Quantitative Finance, Vol. 2, pp. 415431, 2002.

[18] M. Stutzer, "A Simple nonparametric approach to derivative security valuation," Journal of Finance, Vol. 51(5), pp. 1633-1652, 1996.

[19] E. Eberlein, U. Keller, and K. Prause, K., "New insights into smile, mispricing, and Value at Risk: the hyperbolic model,” Journal of Business, Vol. 71, pp. 371-405, 1998.

[20] F. X. Diebold, and R. S. Mariano, "Comparing predictive accuracy," Journal of Business and Economic Statistics, Vol. 13, pp. 253-263, 1995.

[21] E. Renault, "Econometrics of option pricing," in Encyclopedia of Quantitative Finance, Vol. 2, R. Cont, Ed., New York: Wiley, 2010, pp. 518-528. 
[22] O. Barndorff-Nielsen, and N. Shephard, "Non-Gaussian Ornstein-Uhlenbeck-based models and some of their uses in financial economics," Journal of the Royal Statistical Society B, Vol. 63, pp. 167-241, 2001.

[23] L. Benzoni, P. Collin-Dufresne, and R. S. Goldstein, "Explaining asset pricing puzzles associated with the 1987 market crash," Journal of Financial Economics, Vol. 101(3), pp. 552$573,2011$.

[24] R. Jacobs, M. Jordan, and A. Barto, "Task decomposition competition in a modular connectionist architecture: The what and where vision tasks," Cognitive Science, Vol. 15, pp. 219-250, 1991.

[25] J.R. Jang, C. Sun, and E. Mizutani, Neuro-Fuzzy and Soft Computing. NJ: Prentice- Hall, 1997.

[26] J. Bezdek, and N. Pal, "Two soft relatives of learning vector quantization," Neural Networks, Vol. 8(5), pp. 729-743, 1995.

[27] F. X. Diebold, and R.S. Mariano, "Comparing predictive accuracy," Journal of Business and Economic Statistics, Vol. 13, pp. 253-263, 1995. 